\title{
Endoscopic partial reversal of complicated single anastomosis gastric bypass
}

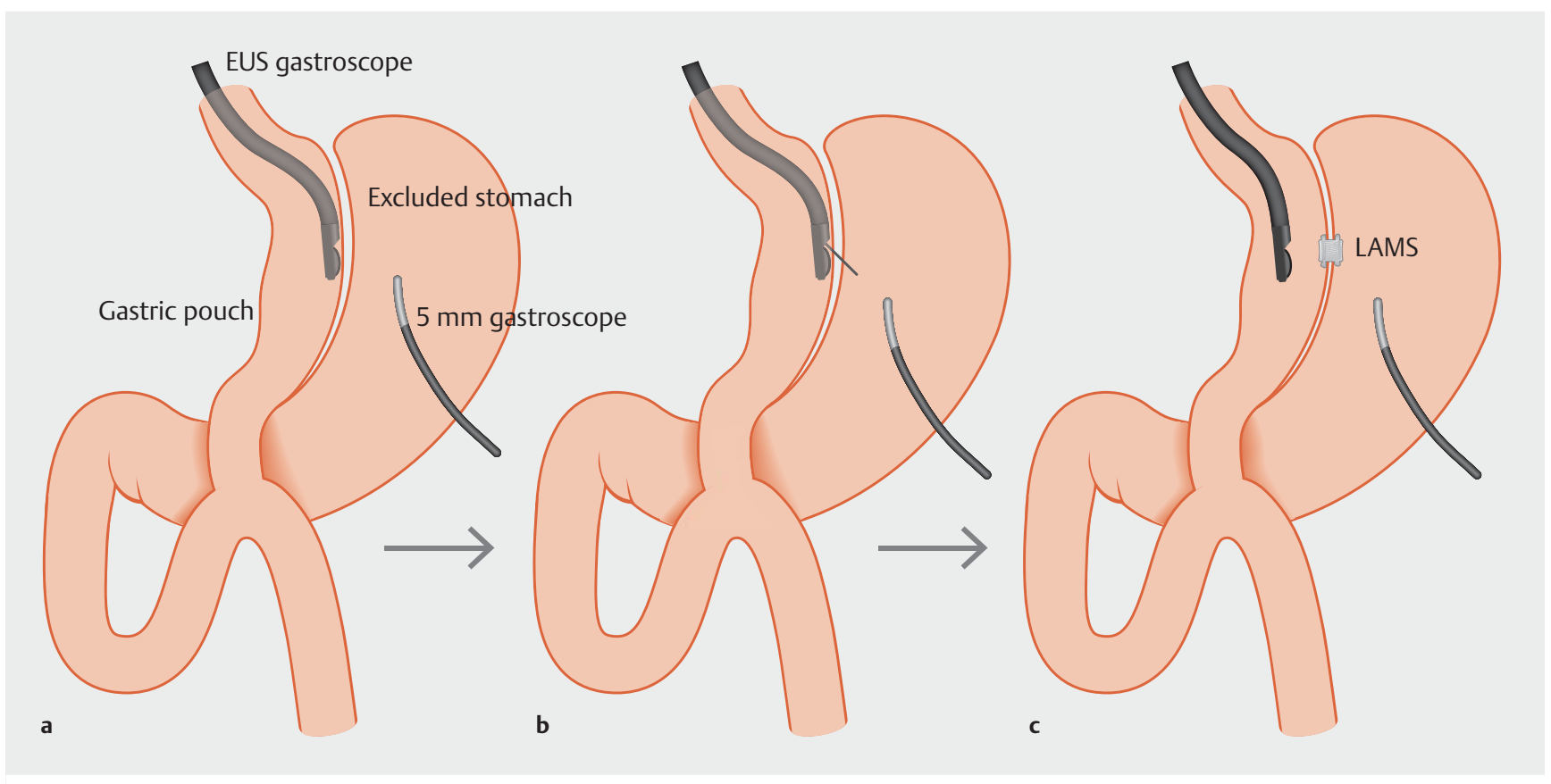

- Fig. 1 Pictorial representation of the procedure. a Endoscopic ultrasound (EUS) and 5-mm endoscope inserted concurrently. b 19-gauge needle puncture from gastric pouch into excluded stomach under EUS guidance. c $15 \times 10 \mathrm{~mm}$ Hot Axios (Boston Scientific, Marlborough, Massachusetts, USA) lumen-apposing metal stent (LAMS) inserted to create gastro-gastric anastomosis.

Prior to the widespread use of therapeutic endoscopy, surgery was the only way to achieve restoration of normal bowel continuity after resection and bypass of a diseased or obstructed gastrointestinal tract. However, with the advancement of endoscopic skills and devices, endoscopic approaches now provide possible alternatives [ $1-5]$. This procedure is particularly useful for patients with hostile abdomen and high operative risk.

A 68-year-old man underwent a laparoscopic single anastomosis or mini-gastric bypass (MGB), with 1.5 metre bilio-pancreatic limb, in 2013 for morbid obesity and poorly controlled diabetes mellitus. Postoperatively, he suffered from severe malnutrition despite increasing oral intake and protein supplements. He was experiencing diarrhea and bilateral lowerlimb edema secondary to hypoalbuminemia $(18 \mathrm{~g} / \mathrm{L})$. He underwent a computed tomography-guided insertion of a gastrostomy tube into the excluded stomach

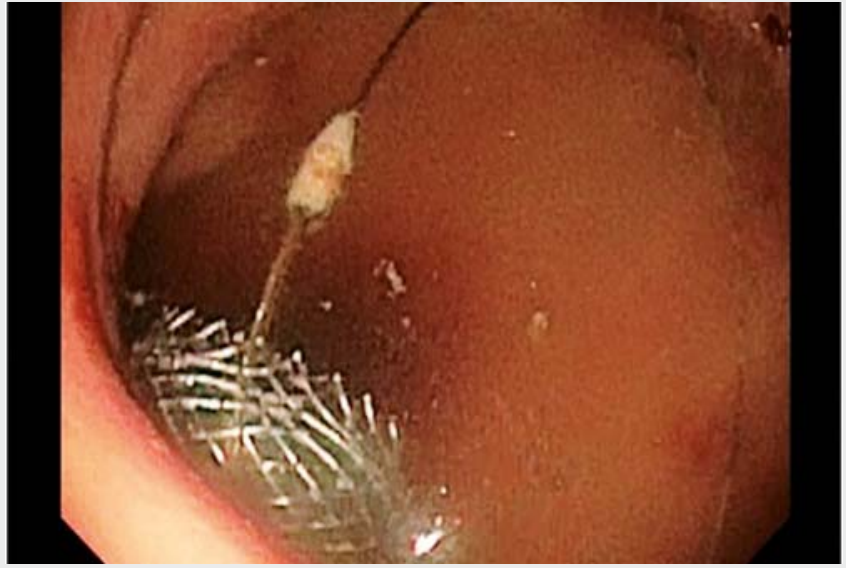

Video 1 Endoscopic partial reversal of single anastomosis gastric bypass. 


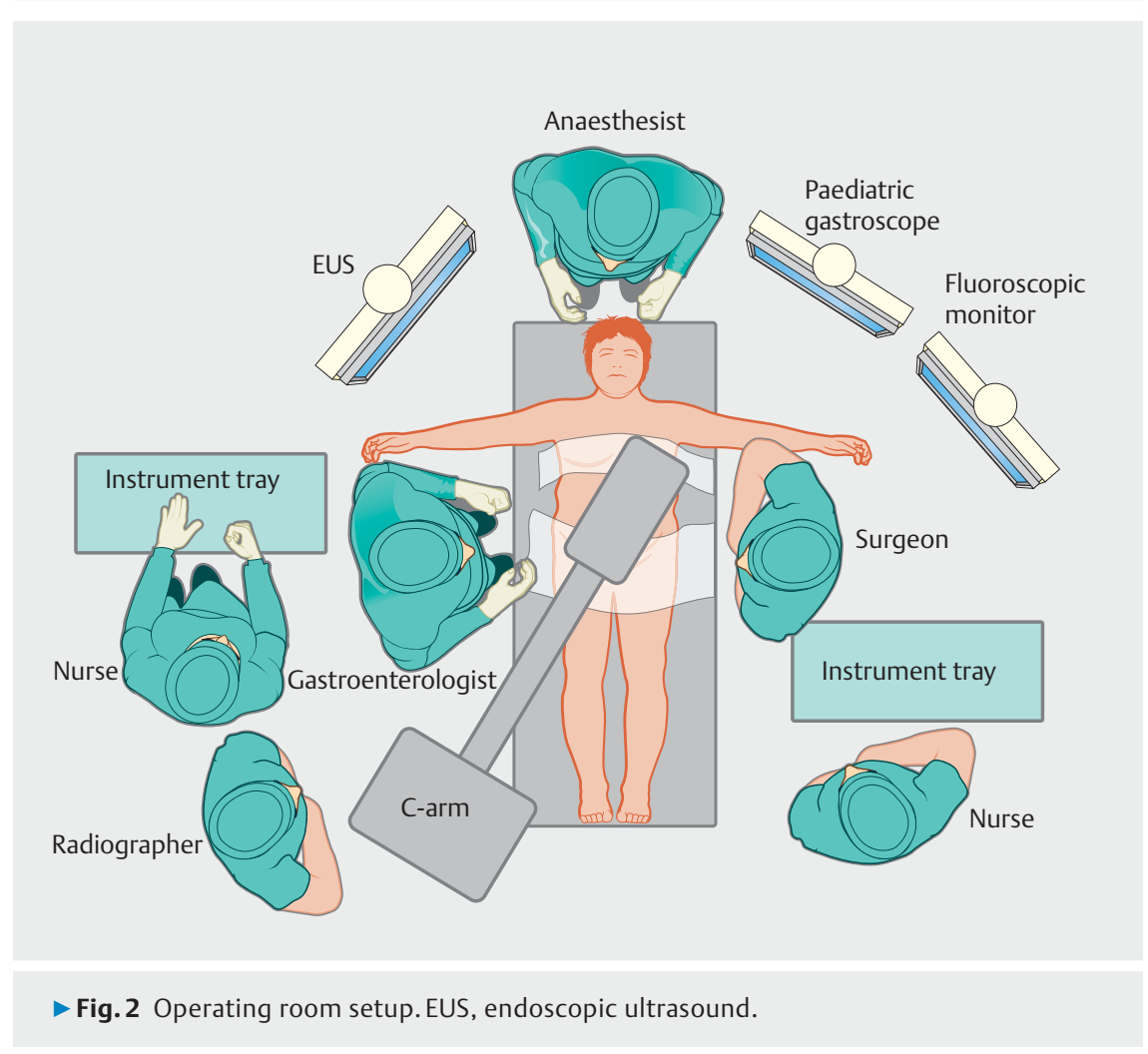

in January 2018 to optimize nutrition by recruiting the excluded foregut. An elective endoscopic partial reversal of MGB ( $\triangleright$ Fig. 1, $>$ Video 1) was then performed 5 months later.

The procedure was performed under general anesthesia. Fluoroscopy was utilized throughout for adjunct imaging. Two operators, each with individual endoscopy towers, were required for the procedure ( $>$ Fig. 2). The first operator inserted a 5-mm gastroscope (GIF-XP160; Olympus, Tokyo, Japan) with a 24-Fr peel-away sheath (MIC-KEY; Halyard, Alpharetta, Georgia, USA) through the gastrostomy tract into the excluded stomach, while an echoendoscope (GF-UC140P-AL5; Olympus) was introduced transorally into the gastric pouch by the second operator. The ideal location for the gastro-gastric anastomosis was selected by using fluoroscopy ( $\mathbf{F i g . 3 a )}$ and endoscopic visualization. A 19-gauge Flex endoscopic needle (Boston Scientific, Marlborough, Massachusetts, USA) was inserted through the echoendoscope to access the remnant stomach and a 0.025-inch VisiGlide guidewire (Olympus) was placed. A $15 \times 10 \mathrm{~mm}$ Hot Axios stent (Boston Scientific) was deployed ( Fig.3b) and dilated to $10 \mathrm{~mm}$ using a balloon (CRE Balloon-Dilator; Boston
Scientific). A pediatric gastroscope was used to confirm satisfactory stent placement ( $\triangleright$ Fig. $3 \mathbf{c}$ ). This procedure took approximately 60 minutes.

At follow-up 2 months later, the patient's weight had increased from $61.8 \mathrm{~kg}$ to $65.8 \mathrm{~kg}$ and albumin levels had increased to $32 \mathrm{~g} / \mathrm{L}$. Contrast study after 3 months showed smooth flow of contrast through both the stent and the anastomosis, at a ratio of 60:40 ( $>$ Fig. 3 d).

Endoscopy_UCTN_Code_TTT_1AO_2AC

Competing interests

None

The authors

Jasmine Hui Er Chang', Damien Meng Yew Tan $^{2}$, Weng Hoong Chan ${ }^{1}$, Alvin Kim Hock Eng', Eugene Kee Wee', Jeremy Tian Hui Tan', Chin Hong Lim ${ }^{1}$

1 Department of Upper Gastrointestional and Bariatric Surgery, Singapore General Hospital, Singapore

2 Department of Gastroenterology and Hepatology, Singapore General Hospital, Singapore

\section{Corresponding author}

\section{Chin Hong Lim, FRCS}

Department of Upper Gastrointestinal and Bariatric Surgery, Singapore General Hospital, Academia, 20 College Road, Singapore, 169856

Fax: +65-62209323

lim.chin.hong@singhealth.com.sg 

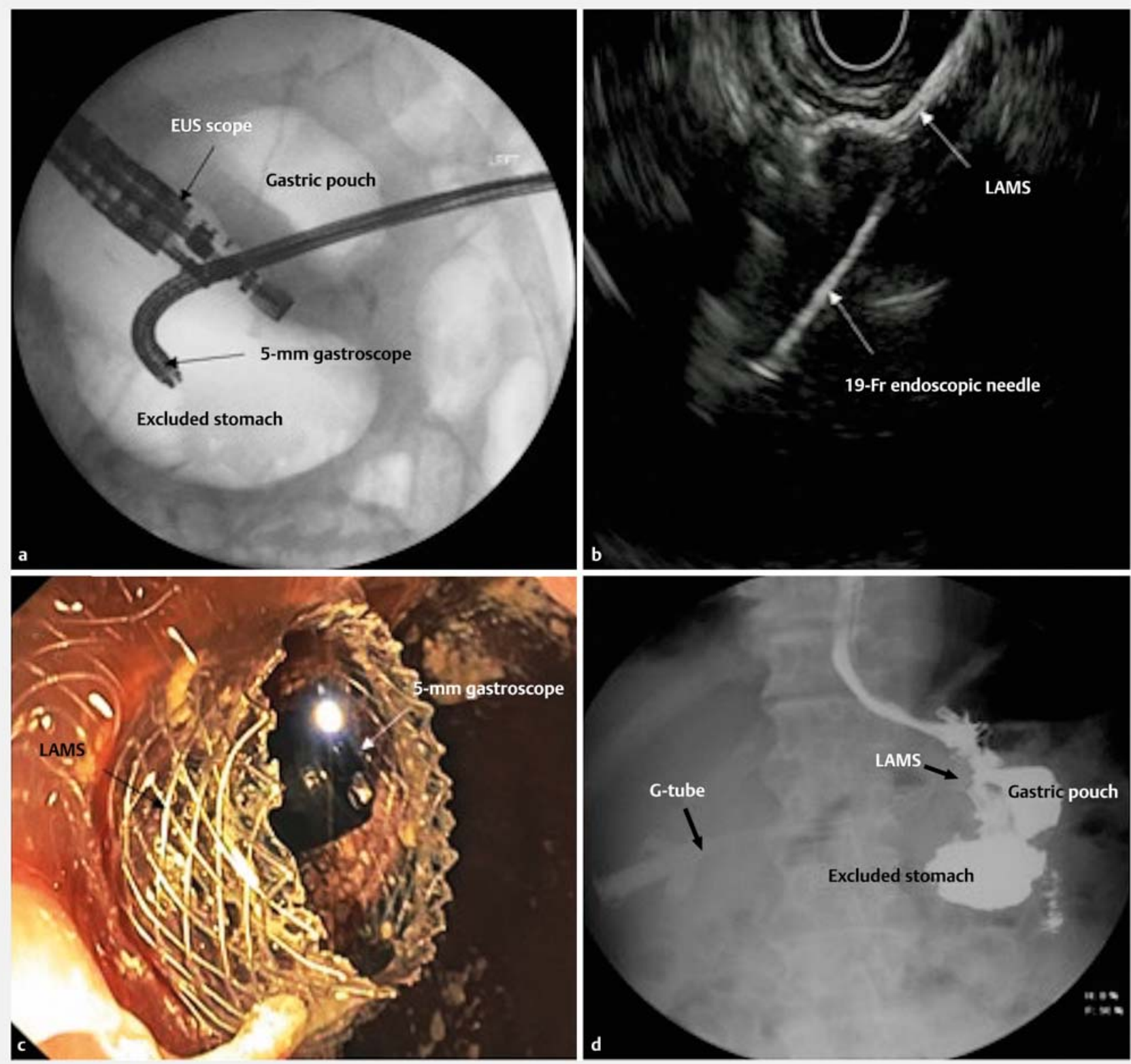

Fig. 3 Graphical representation of the procedure. a Fluoroscopic view: echoendoscope (EUS scope) and 5 mm endoscope were inserted concurrently. b A 19-gauge needle was used to puncture the gastric pouch into the excluded stomach under EUS guidance. c A $15 \times 10$ mm Hot Axios (Boston Scientific, Marlborough, Massachusetts, USA) lumen-apposing metal stent (LAMS) was inserted to create a gastro-gastric anastomosis. d Contrast study 3 months later showed smooth flow of contrast through the stent and gastrojejunostomy.

\section{References}

[1] Amateau SK, Lim CH, McDonald NM et al. EUS-guided endoscopic gastrointestinal anastomosis with lumen-apposing metal stent: feasibility, safety, and efficacy. Obes Surg 2018; 28: $1445-1451$

[2] Tyberg A, Perez-Miranda M, Sanchez-Ocana R et al. Ultrasound-guided gastrojejunostomy with a lumen-apposing metal stent: a multicenter, international experience. Endosc Int Open 2016; 4: E276-E281
[3] Khashab MA, Kumbhari V, Grimm IS et al. EUS-guided gastroenterostomy: the first U.S. clinical experience. Gastrointest Endosc 2015; 82: $932-938$

[4] Itoi T, Ishii K, Ikeuchi N et al. Prospective evaluation of endoscopic ultrasonographyguided double-balloon-occluded gastrojejunostomy bypass (EPASS) for malignant gastric outlet obstruction. Gut 2016; 65: 193 195

[5] Lim CH, Leslie D, Ikramuddin S et al. Novel endoscopic management of a pancreatobiliary limb obstruction by the creation of an entero-enterostomy following Roux-en-Y gastric bypass. Endoscopy 2016; 48: E99E100

\section{Bibliography}

DOI https://doi.org/10.1055/a-0841-9831

Published online: 21.2.2019

Endoscopy 2019; 51: E108-E110

(C) Georg Thieme Verlag KG

Stuttgart · New York

ISSN 0013-726X 